\title{
Necesidades de los familiares de pacientes con esquizofrenia en situaciones de crisis
}

\author{
María Luisa Rascón, ${ }^{1}$ Marcelo Valencia, ${ }^{1}$ Tecelli Domínguez, ${ }^{2}$ Humberto Alcántara, ${ }^{3}$ Leticia Casanova'
}

Artículo original

\section{SUMMARY}

\section{Objetive}

This article presents the main crisis situations faced by the relatives of schizophrenic patients, as well as their needs in psychiatric attention at moments of crisis.

\section{Method}

Qualitative study. A discussion group of eight parents of patients with schizophrenia was conformed. The group sessions were recorded and transcribed to analyze the information later on. In addition, the information was complemented with individual interviews of the relatives.

\section{Results}

Parents emphasized three main crisis situations: 1. a first psychotic crisis, 2. family crisis facing the diagnosis and in the process of acceptance of the disease, and 3. subsequent relapses. 4. Deficiencies in the care crisis, and 5. needs of families in crisis situations are also detailed.

\section{Conclusions}

According to the narratives related by the relatives of the discussion group, we can conclude that it is necessary to provide information to the family, the patients and the general population, so that there is a knowledge of the main characteristics and symptoms of schizophrenia. This should be carried out with the purpose of obtaining an early detection that facilitates the treatment and prevents later crises interventions and creating a social support network in the community.

Key words: Needs' crisis, schizophrenia, family, qualitative study.

\section{RESUMEN}

\section{Objetivo}

Identificar y analizar las principales situaciones de crisis que enfrentan los familiares de pacientes con esquizofrenia, así como sus necesidades en la atención psiquiátrica en momentos de crisis.

\section{Método}

Con metodología cualitativa de recolección y análisis, la técnica utilizada fue la creación de un grupo de discusión con ocho familiares de pacientes con esquizofrenia. Las sesiones grupales se grabaron y transcribieron para analizar posteriormente la información. Además, se realizaron entrevistas individuales a cada familiar.

\section{Resultados}

Los familiares destacaron tres principales situaciones de crisis: 1. La crisis psicótica; 2. La crisis familiar ante el diagnóstico y en el proceso de aceptación de la enfermedad; 3 . La crisis ante las recaídas subsecuentes. También se detallan: 4. Las deficiencias en la atención en situaciones de crisis y 5 . Las necesidades de los familiares en estas situaciones.

\section{Conclusiones}

Desde la narrativa de los familiares se puede destacar la experiencia de una falta de información tanto a familiares y a pacientes como a la población en general, para que se conozcan los principales rasgos y síntomas que caracterizan a la esquizofrenia, para lograr con ello una detección temprana que facilite el tratamiento y la prevención de crisis posteriores. Es conveniente desarrollar intervenciones en los momentos de crisis y establecer una red social de apoyo en la comunidad.

Palabras clave: Necesidades, crisis, esquizofrenia, familia, estudio cualitativo.

\section{INTRODUCCIÓN}

La Organización Mundial de la Salud (2010) estima que en todo el mundo alrededor de 29 millones de personas padecen esquizofrenia. Aunque su incidencia es baja (3 por cada
$10000)$, su prevalencia es alta debido a la cronicidad de esta enfermedad. ${ }^{1}$ Se calcula que una de cada cuatro personas se ve afectada por un trastorno mental grave en algún momento de su vida. Lo anterior implica que, por cada diez personas con trastorno mental grave, hay 2.5 cuidadores. $^{2} \mathrm{El}$

\footnotetext{
Dirección de Investigaciones Epidemiológicas y Psicosociales, INPRFM. Facultad de Medicina, Universidad Nacional Autónoma de México.

2 Departament de Psicologia Clínica i de la Salut, Universitat Autònoma de Barcelona, España.

Dirección de Investigaciones Epidemiológicas y Psicosociales, INPRFM.
}

Correspondencia: María Luisa Rascón Gasca. Dirección de Investigaciones Epidemiológicas y Psicosociales, Instituto Nacional de Psiquiatría Ramón de la Fuente Muñiz, Calz. México-Xochimilco 101, San Lorenzo Huipulco, Tlalpan, 14370, México DF. E-mail: rascong@imp.edu.mx

Recibido: 13 de noviembre de 2013. Aceptado: 16 de enero de 2014. 
cuidado de una persona que padece el trastorno plantea una serie de desafíos importantes en el entorno familiar. El apoyo del cuidador es esencial para la estabilidad de la persona con esquizofrenia, ya que puede ayudar a prevenir las posibles crisis psicóticas y puede contribuir a mejorar el nivel de funcionamiento global y su apego terapéutico. Asimismo, uno de cada dos familiares de personas con esquizofrenia está en riesgo de presentar algún trastorno emocional, físico o psiquiátrico relacionado con la carga de ser el familiar cuidador principal informal. ${ }^{3}$

El concepto de crisis tiene diversas connotaciones y tiende a asociarse con la ruptura del equilibrio o de la "normalidad", o con la aparición o reaparición súbita de un trastorno. ${ }^{4}$ Esta situación de cambio implica un desafío y una reorganización para enfrentar los hechos que la desencadenan.

Vírseda ${ }^{5}$ define crisis como el estado psicológico resultante del enfrentamiento de la familia con hechos percibidos como peligrosos, acompañados de sentimientos de incapacidad para resolver la situación de forma eficiente con los recursos con que se cuenta en el momento.

Existen estudios sobre la intervención y la prevención de crisis en pacientes con esquizofrenia, ${ }^{6-8}$ sobre la importancia del papel que cumple la familia en el tratamiento y sobre las consecuencias que provoca la esquizofrenia en los parientes del enfermo. ${ }^{9-18}$ Sin embargo, pocos estudios toman en cuenta los problemas que enfrentan los familiares cuando necesitan atención en momentos de crisis.

El presente artículo tiene como objetivo dar a conocer la experiencia y la narrativa de las principales situaciones de crisis que enfrentan los familiares de pacientes con esquizofrenia, así como sus necesidades en la atención psiquiátrica en momentos de crisis.

\section{MÉTODO}

Estudio de corte cualitativo basado en la experiencia de grupos de discusión ${ }^{19}$ de familiares de personas con esquizofrenia. El muestreo fue a conveniencia de casos tipo. Se invitó a familiares que acudían a instituciones públicas de salud mental en la Ciudad de México. El criterio de inclusión fue tener un familiar con esquizofrenia con el cual convivían cotidianamente; las personas con esquizofrenia recibían en el momento del estudio tratamiento integral (psiquiátrico y psicosocial).

La cooperación fue voluntaria, anónima y confidencial. Se obtuvo el consentimiento informado de los participantes, según el cual estuvieron de acuerdo en participar en las sesiones grupales sabiendo que éstas serían grabadas. El estudio fue dictaminado previamente por un comité de ética y cumplió con la normatividad internacional de bioética.

La investigación consistió en crear un espacio de diálogo construido en cada sesión y permitió hacer fluir información que no solamente profundiza en los temas que genera, sino que amplía el bagaje informativo mediante el efecto sinérgico y la interrelación del grupo. ${ }^{20}$

Se tomó en cuenta la teoría narrativa que examina cómo las personas atribuyen sentido a su experiencia y su existencia. ${ }^{21}$ Esta teoría sugiere que la integración de lo subjetivo y lo objetivo de las estrategias de investigación da como resultado una comprensión más completa de la esquizofrenia. Además, se entrevistó individualmente a cada uno de los familiares para complementar la información.

\section{Procedimiento y análisis de la información}

Las sesiones grupales del presente estudio fueron guiadas por una serie de aspectos que permitieron abordar diversos temas como: la significación de la esquizofrenia, la experiencia y las principales necesidades de los familiares ante las situaciones de crisis a las que se han enfrentado a lo largo del proceso de la enfermedad. El proceso de análisis se efectuó a partir de las transcripciones de las dos sesiones grupales. En primer lugar se obtuvieron las principales categorías de análisis o ejes temáticos. En segundo lugar se seleccionaron algunos fragmentos de las narrativas de los familiares que se consideraban representativos de la temática del presente estudio, para finalmente articular dicha información mediante contenidos teóricos.

\section{RESULTADOS}

\section{Descripción demográfica de los familiares}

El grupo estuvo conformado por ocho familiares (siete mujeres y un hombre), de los cuales seis fueron madres, una hermana y un padre, con un promedio de edad de 59.44 años. El $44.4 \%$ tenía pareja y el $55.6 \%$ no la tenía; el $33.3 \%$ estudió preparatoria y 55.6\% estudió uno o más años de la universidad. Al momento del estudio, el $77.8 \%$ tenía una actividad remunerada y el $22.2 \%$, no remunerada (se dedicaban al hogar). El $44.4 \%$ de los familiares convivía con su familiar enfermo todo el día, y el resto (55.6\%) lo veía a ratos por la mañana, la tarde o la noche. En relación con las recaídas con hospitalización de los pacientes, el 33.3\% de ellos no había recaído, el 22.2\% había tenido al menos una recaída con hospitalización, el 33.3\% había recaído dos veces y, el $11.2 \%$ había recaído tres veces.

\section{La primera crisis psicótica}

Los familiares que participaron en el grupo de discusión coincidieron en que la aparición de estos comportamientos en sus hijos fue perceptible antes de que les diagnosticaran la esquizofrenia y, en la mayoría de los casos, esta fase coincidió con la etapa de la adolescencia. Incluso una familiar mencionó que los primeros síntomas que presentó su hijo 
se confundieron con las conductas típicas de los adolescentes:

F7: "Como se le presentó a los 19 o 20 años, yo creía que era una cosa de la adolescencia, de esa flojera, pues, como se comportan los adolescentes."

F5: "[...] Saliendo de la prepa fue cuando se empezó a aislar, empezó a pensar que la gente que venía a visitarlo le quería hacer daño [...] y así, yo lo veía como ausente, sus ojos de repente parpadeaban y se le veían como en blanco, o sea, cosas muy raras. Yo decía, ¿qué le está pasando?, no lo comprendía."

F1: "Mi hijo, él de repente, él trabajaba, él era verdaderamente muy capaz en el trabajo y todo. Después, de un accidente [...]. Posteriormente, él iba subiendo las escaleras y azotó en la escalera; yo me asusté muchísimo y le hablé a mi doctor, [...] y le pregunté, ¿qué tiene? y me dijo, pues nosotros tenemos la sospecha de que él pudiera tener un problema mental [...] y yo no entendía [...]."

F2: "Vivía yo en la ignorancia total y ya gracias a Dios ya tengo información, ya lo diagnosticaron y como que ya empecé a respirar, pero tardé mucho tiempo desesperada, pasé mucho tiempo desesperada [...]."

Se ha mostrado que los servicios especializados de atención a la salud mental se utilizan poco y en su lugar se recurre a médicos generales, familiares, amigos y/o religiosos. Se ha manifestado también que un importante porcentaje de la población que recurre al primer nivel de atención presenta trastornos mentales, pero que éstos son detectados pocas veces y mucho menos referidos a tiempo. ${ }^{22}$ Como consecuencia, los familiares van de un lugar a otro y pasan por muchas instituciones antes de recibir la atención que necesitan. El siguiente fragmento es un ejemplo de esta situación:

F2: "[...] en un principio me fue muy difícil manejar la situación porque nunca tuve una información directa. Primero empecé a ver a la psicóloga del colegio de mi hijo, porque mi hijo se había peleado en dos ocasiones y ya a la tercera había agredido a la maestra. La psicóloga me sugirió una terapia familiar; no logré esa terapia familiar. [...] Esa psicóloga me mandó con un psiquiatra. [...] Él nunca me dijo 'vamos a hacer un estudio, vamos a hacer una investigación', ;nada!. Sí se le hizo un encefalograma [...] y la cosa es que a la larga nunca oí la palabra esquizofrenia."

Hoy en día, el intervalo de tiempo que transcurre desde los primeros síntomas diagnosticables de esquizofrenia hasta el momento en que es diagnosticado el paciente oscila en general entre uno y dos años. ${ }^{23,24}$ A este tiempo se le debe sumar el retraso que se produce hasta que se inicia el tratamiento. De ahí la importancia de entrenar mejor a los médicos generales y psicólogos para que dispongan de herramientas que aseguren el diagnóstico precoz y la derivación del paciente a una atención en salud mental en el momento oportuno. ${ }^{24}$

Es frecuente que los familiares no busquen ayuda sino hasta que el paciente está en crisis psicóticas, presenta actitudes muy extrañas, tiene un comportamiento violento difícil de controlar o está en situaciones de riesgo. Todo lo anterior genera un conflicto, porque la mayoría de las veces estos rasgos hacen que el paciente tenga que ser hospitalizado. ${ }^{25}$

F7: "Al principio pensé que era la adolescencia, pero se fue complicando la situación. Hasta que un día una compañera me habló y me dijo que si no me había dado cuenta que mi hijo hacía cosas peligrosas y entonces fue cuando nos empezamos a preocupar porque se ponía a decir y a hablar a la gente cosas, ideas y fuera de lugar; entonces se cruzaba la calle con los brazos abiertos, cosas muy raras. Entonces [...] fuimos directamente con un psiquiatra [...]."

La falta de información provoca que los pacientes y sus familiares acudan al médico hasta que se presenta una crisis o el primer episodio psicótico, momento en que el impacto es mayor y el manejo es más complejo, en vez de buscar atención psiquiátrica en esa fase prodrómica cuando los síntomas pueden servir para prevenir la crisis. En este sentido, la importancia de detectar y tratar oportunamente a los pacientes con primer episodio psicótico radica en prevenir el desarrollo de alteraciones neurobiológicas que compliquen el pronóstico. ${ }^{26}$

Hay que tomar en cuenta que, además de la desinformación generalizada acerca de la esquizofrenia, hay otros aspectos que demoran la búsqueda de apoyo profesional. Por un lado está el estigma que hay hacia el paciente psiquiátrico que se asocia con "estar loco"; por otro lado está la negación de la enfermedad y el impacto que causa el diagnóstico en los familiares, lo cual puede considerarse como otro tipo de crisis, como se verá a continuación.

\section{Crisis familiar ante el diagnóstico y en el proceso de aceptación de la enfermedad}

En la familia, las relaciones y su dinámica se modifican cuando se introduce en la misma el requerimiento de la atención psiquiátrica. Una de las familiares del grupo describió la esquizofrenia como "un trastorno familiar":

\section{F7: "[La esquizofrenia] arrastra a la familia."}

La esquizofrenia puede representar una doble problemática: por un lado, atender los problemas de la enfermedad por sí misma y, por otro, superar las condiciones sociales adversas que se generan en torno a ésta. A partir de que surge la esquizofrenia la dinámica familiar se transforma y la mayoría de las veces es difícil aceptar el hecho de que un familiar padezca una enfermedad mental:

F1: "[...] Muchos decimos a mí no me va a pasar; entonces, en ese instante, ejerces rechazo."

F7: "[...] Cuando el psiquiatra nos dijo que era una cosa muy seria, no me imaginé qué era, hasta después que dijo que era esquizofrenia y como mi esposo tiene una hermana que también tiene, pues dije: jno puede ser, no!."

F6: "Estaba consciente de que había un problema, pero no aceptaba de ninguna manera que él tenía eso, o sea, yo no sabía ni que aunque el doctor me lo explicaba, hasta hacía mímicas y gestos. El otro doctor, el psiquiatra, yo le decía no es cierto, no es cierto, no es cierto."

En un primer momento, el paciente y sus familiares niegan la enfermedad porque el diagnóstico genera un gran impacto y una tensión constante, lo cual, en muchos casos, dificulta a su vez la adherencia al tratamiento. ${ }^{27}$

F1: "[...] No hay aceptación de por sí, para uno es difícil aceptar que el familiar de uno, en mi caso mi hijo, tenga esta enfermedad. Cuando 
se le manifiesta por alguna razón a los familiares, la primera reacción es negarlo, jno! ¿Cómo crees?"

F4: "Yo creo que otro obstáculo es la propia familia, que no acepta que está enfermo, te dicen que te chantajea, que en realidad la persona no está enferma, entonces también eso dificulta mucho."

La experiencia de enfrentar el diagnóstico cuando no se tiene información suficiente genera, por una parte, angustia y desesperación en los familiares, y por otra, produce mucha incertidumbre en cuanto al tratamiento y las expectativas de vida del paciente. Es por esto que la atención es necesaria porque el diagnóstico provoca un daño emocional:

F6: "No podemos estar solos y aislados ante un diagnóstico que nos hace daño. Emocionalmente nos hace daño, porque mi hijo no puede tener eso [...] Estoy enojado con todos y por eso necesito atención, simplemente tengo un familiar que tiene ese problema."

El médico tiene que tomar en cuenta que el diagnóstico genera un bloqueo emocional en los familiares que les impide escuchar claramente las explicaciones iniciales; por ello, es necesario concertar otras consultas para cerciorarse, por medio de preguntas, de que la familia ha entendido. A pesar de que resulta tedioso para el médico - y posiblemente costoso para las instituciones - , el tiempo que se invierta en estas primeras sesiones tendrá sus frutos en el transcurso del tratamiento, pues con ello se evitarán muchos errores y malas interpretaciones. ${ }^{28}$

El proceso de aceptación de la enfermedad y la forma en que cada miembro de la familia se involucra en el tratamiento del paciente son distintos, según el tipo de relación que se mantiene con el enfermo y la forma de afrontar la situación. ${ }^{13}$ Algunos familiares tienen que dejar su trabajo para hacerse cargo del paciente, mientras que otros simplemente no soportan la situación familiar y evitan la convivencia, como se ejemplifica a continuación:

F7: "Inclusive mi hija, ella se fue a Inglaterra, como huyendo de la situación familiar, y ella me comentaba siempre que mi hijo no servía, que no iba a servir para nada. Y a mí me dolía mucho eso, pero tenía razón ella en expresar lo que sentía, porque lo veía siempre acostado sin seguir en la escuela ni conseguir un trabajo."

Al estudiar a los familiares cuidadores se encontró que más de la mitad (58.8\%) de ellos presentaron algún trastorno psiquiátrico, dentro de los cuales se destacaron el diagnóstico de depresión en las mujeres y la dependencia al alcohol en los hombres. ${ }^{3}$ Esto puede ser consecuencia de la situación de constante estrés que se vive en la familia por la esquizofrenia y del hecho de que algunos familiares se descuiden personalmente debido al cuidado y la sobreprotección que le dan a su familiar enfermo. Algunos estudios han reportado que uno de cada tres familiares cuidadores presenta niveles elevados de ansiedad o depresión vinculados con su papel de cuidador. ${ }^{29} \mathrm{El}$ siguiente fragmento da cuenta de las consecuencias que puede tener en una madre el descuido personal por la dedicación total hacia el enfermo:

F3: "Dos años que me entregué así, ipum! Y cuando después ya lo levanté de la bronca porque lo solté, pero también vino para mí, que de pronto [...] hubo que voltear hacia mí porque me vino una depresión pavorosa."

\section{Las recaídas subsecuentes y las continuas crisis}

El 66.6\% de los participantes del grupo de discusión habían tenido la experiencia de recaídas en sus familiares enfermos.

Lader $^{30}$ define la recaída como el retorno de una enfermedad después de una recuperación total o parcial. La recaída en pacientes con esquizofrenia puede ser evaluada por medio de los niveles sintomáticos, fenomenológicos y conductuales. Se detecta a partir de la aparición o el resurgimiento de síntomas psicóticos como delirios, alucinaciones, comportamientos extraños o trastornos del pensamiento.

En algunos estudios se ha observado que $80 \%$ de los pacientes diagnosticados con un primer episodio de esquizofrenia o trastorno esquizoafectivo presentan en promedio dos recaídas en los primeros cinco años de evolución. ${ }^{31}$ Las razones por las que un paciente recae son muy diversas, aunque se ha reportado que el índice de recaídas es mayor en los pacientes que sólo reciben tratamiento farmacológico en comparación con aquellos que recibieron tratamiento psicosocial y psicoeducación en conjunto con el tratamiento farmacológico. ${ }^{15}$ Sin embargo, también se ha visto que muchos pacientes con esquizofrenia reciben medicación en las dosis preescritas y a pesar de eso presentan recaídas. ${ }^{27}$

Las recaídas que mencionan los familiares que participaron en el grupo de discusión fueron provocadas por mal manejo o retiro de medicamentos.

F8: "[...] sólo ha tenido dos internamientos cuando se le diagnosticó y cuando le dio la artritis y fue lo mismo, le quitaron el medicamento y por eso recayó [...] Llegó a consulta, le bajó la dosis casi a la mitad y mi hermana recayó hace quince días."

F5: "Hubo un problema que es la única recaída que ha tenido mi hijo en todo el transcurso de su enfermedad, que fue que lo internaron [...] Pues al otro día le falló el medicamento, [el doctor] le quitó de golpe todo el medicamento [...]."

Hay estudios donde se establece que la principal variable de la recaída es la suspensión del tratamiento, ya que, con el retiro de la medicación, el $67 \%$ de los pacientes recaen en el transcurso de un año. ${ }^{26}$

También existen estudios recientes enfocados en la Emoción Expresada familiar (EE), considerada como el mejor predictor de recaídas que se conoce actualmente para personas con esquizofrenia, y que podría caracterizarse como un estilo comunicativo del familiar con el paciente que influye en el curso de la enfermedad. ${ }^{24}$

El ambiente familiar y las actitudes básicas hacia el paciente ejercen una poderosa influencia en su calidad de vida percibida, incluso por encima de otras variables como la psicopatología. ${ }^{17,32,33}$ Así, los enfermos con una familia de alta EE tienen hasta cuatro veces más probabilidades de recaer que los que tienen una familia de baja EE. ${ }^{24}$ 
La predicción de las recaídas en esquizofrenia es un componente importante que hay que considerar, porque hay evidencia que indica que casi la mitad del 78\% de los pacientes con esquizofrenia ha tenido episodios repetidos y experimenta un deterioro progresivo en el funcionamiento después de cada recaída sucesiva. ${ }^{34}$

Consideramos que una recaída representa una crisis porque es una situación imprevista y grave donde hay un cambio radical en el comportamiento del paciente (debido a la aparición de síntomas), que implica tomar decisiones. Asimismo, muchas veces, una situación de riesgo puede desatar un intento o consumación del acto suicida.

Después de conocer las diversas situaciones de crisis a las que se enfrentan los familiares, mencionaremos las deficiencias y necesidades en la atención.

\section{Las deficiencias en la atención a situaciones de crisis}

Todos los participantes coinciden en que hay una serie de deficiencias en la atención de los servicios de urgencias. Uno de los problemas que comentaron fue que, frente a la cantidad de la demanda, las citas con el psiquiatra son muy espaciadas, y que cuando sus familiares enfermos tienen una crisis o recaída antes de su cita, tienen que acudir a urgencias. Ahí, según su experiencia, la atención que han recibido ha sido deficiente, debido a la falta de personal capacitado que no tiene suficiente experiencia porque están en proceso de formación. También resulta paradójico que los psiquiatras más capacitados no atiendan a aquellos pacientes en crisis que necesitan atención urgente.

F8: "No puedo acudir al servicio de urgencias porque a la residente le falta criterio y el médico general va saliendo del primer año [...] ni siquiera la recibió [...], yo creo que les falta mucho [...]."

F4. "Me preocupa mucho que mi hijo se requiera internar y no haya cama para hospitalizarlo."

La crisis, como vimos anteriormente, es una situación inesperada y repentina que difícilmente se suscita en la fecha exacta de la cita con el psiquiatra. Por ello, es que, en momentos de crisis, los pacientes casi siempre tienen que llegar a urgencias para ser tratados.

Los pacientes en crisis que acuden a urgencias y que están lo suficientemente graves son hospitalizados, mientras que aquellos que no ameritan ser internados son enviados de regreso a su casa, sin recibir la atención merecida, ni información adecuada para que sus familiares sepan qué hacer si se presenta otra crisis. Esta situación es difícil porque, por un lado, la familia que busca ayuda en una crisis está preocupada porque su familiar puede requerir un internamiento.

F8: "Llegó a consulta, le bajó la dosis casi a la mitad y mi hermana recayó hace quince días. Fui a urgencias; la residente me dijo: 'no, tenemos saturada la consulta del doctor, todos vienen a urgencias, su hermana no está mal'. Ni siquiera la revisó, ni la bajé de la camioneta, me dio una receta y me dijo 'tráigala con la doctora'. ¡Pero es que estamos en marzo y le toca cita hasta mayo!"
Por otro lado, los familiares coincidieron en que a algunos médicos del servicio de la consulta les hace falta tacto, empatía y atención en situaciones de urgencia para detectar posibles recaídas.

F2: "[La doctora] no me contesta, en una de esas dos ocasiones le he marcado hasta quince veces durante tres días o cuatro y no logro hablar con ella. Entonces me desmoralizo, me decepciono en tal forma que me ahoga esa falta de atención. Porque no tengo a quién más llamar, porque nadie más conoce a [mi hijo] puesto que ella es su médico."

\section{Necesidades de los familiares en la atención a crisis}

Después de conocer las diversas situaciones de crisis a las que se enfrentan, consideramos relevante tomar en cuenta las necesidades en situaciones de crisis que mencionaron los participantes del grupo.

Necesidad de información, apoyo profesional e institucional para el manejo de crisis

Los familiares coincidieron en que hace falta informar a la población en general sobre los trastornos mentales para que tenga conocimiento de los principales síntomas y para que se pueda lograr una detección temprana que prevenga crisis posteriores (figura 1).

F2: "En un principio me fue muy difícil manejar la situación, porque nunca tuve una información directa."

F6: "No hay información clara, no hay información oportuna."

F2 "Información para que la gente sepa a dónde pueden ir cuando se presenta una crisis. Esta información debe ser a nivel masivo, puesto que nunca se sabe quién puede presentar una crisis psicótica y es muy difícil saber qué hacer."

También es importante que se difunda información a través de los medios de comunicación acerca de las diferentes instituciones y hospitales encargados de atender los trastornos mentales, en los cuales buscar ayuda cuando no se tiene la información adecuada para tomar una decisión en una situación de crisis, sobre todo si es la primera vez que se presenta la sintomatología psicótica.

Cuando el paciente ya tiene un diagnóstico de esquizofrenia, el tipo de información que se brinde debe ser más específica y detallada tanto para el familiar como para el usuario, entender qué es la esquizofrenia, cómo se trata, qué consecuencias tiene, cuál es el pronóstico, etc. Esta falta de conocimiento conlleva diversas complicaciones que dificultan el manejo de la enfermedad como: la autopercepción de una capacidad limitada para enfrentar las crisis, el establecimiento inadecuado de expectativas claras y objetivas y el incumplimiento de estas expectativas por parte del enfermo. ${ }^{15}$

F6: "La enfermedad es impactante para el familiar y cuando al familiar se le trata sin información [...] yo creo que le explican más a alguien que tiene cáncer que a alguien que tiene esquizofrenia. Entonces si la enfermedad es tan impactante para la familia, ¿por qué somos tratados así?, sin más información, o sea, necesitamos información clara $[\ldots]^{\prime \prime}$ 


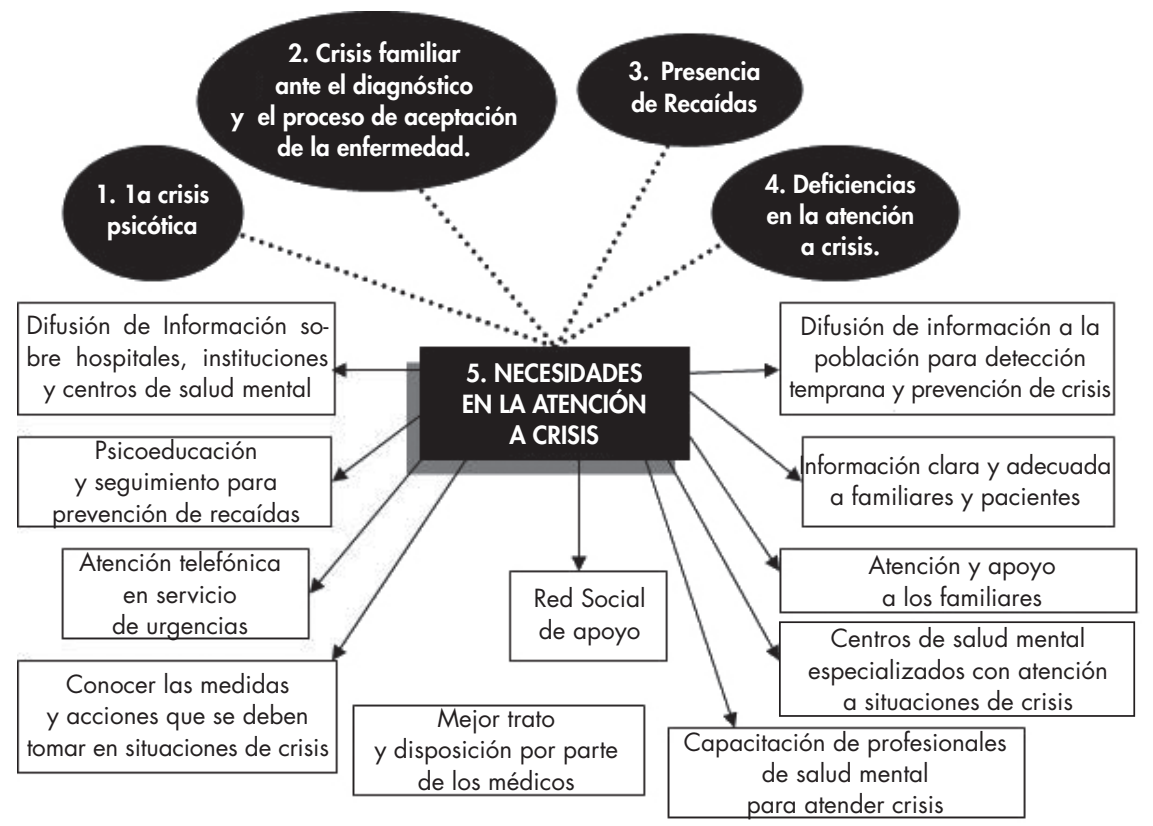

Figura 1. Experiencias y necesidades en situaciones de crisis de los familiares.

Es trascendente que en las diferentes instituciones de salud mental brinden y difundan sistemáticamente información acerca de los síntomas, la etiología y el tratamiento, con el objetivo de mejorar el entendimiento y las conductas asociadas a la enfermedad. Con ello se busca disminuir el índice de recaídas y mejorar la calidad de vida del paciente. ${ }^{10}$

Así como la familia es un factor muy relevante en el tratamiento de la esquizofrenia, es necesario que reciba una atención más allá de la información y capacitación, porque todos los miembros de la familia resultan afectados por la enfermedad y la mayoría de las veces requieren ser escuchados para aligerar el impacto y la tensión que produce la convivencia con su familiar enfermo.

\section{Necesidad de atención integral}

Los participantes del grupo también revelaron la necesidad de una atención integral (tratamiento farmacológico, psicoterapia individual, grupal y familiar, terapia ocupacional, psicoeducación, etc.), ya que están implicados en la problemática de la enfermedad y se ven afectados por la carga que conlleva el cuidado de su familiar enfermo.

F6: "[...] es que la familia necesita una atención integral, o sea, la esquizofrenia no es una cosa aislada de mi hijo, la esquizofrenia tiene que ver con sus hermanos, tiene que ver con mi esposa y conmigo; y con los familiares de ella y míos. O sea, la esquizofrenia está involucrada alrededor de mi hijo que está enfermo, está involucrando a todos los que están alrededor y tratar únicamente con los familiares directos esta enfermedad no pasa de ahí, no resuelve, no resuelve el problema."

Existen estudios sobre el tratamiento integral ${ }^{15-17}$ que demuestran una notable mejoría en el autocuidado, la socialización, el apego terapéutico y en la mejoría de las re- laciones familiares que normalmente están alteradas en el paciente con esquizofrenia.

En la medida en que los familiares tengan un espacio donde sean informados y escuchados y reciban el apoyo profesional y de pares adecuado, será más fácil "lidiar" con la enfermedad cuando se presenten situaciones de crisis.

F7: "Crecer nosotros, fortalecernos nosotros para poder lidiar con las situaciones que se presentan."

F4: "Someterse uno a terapia, buscar la ayuda, ¿no?, de los especialistas, tanto para ella como para la familia. Informar también a la familia."

Más allá del tratamiento farmacológico exclusivo, el seguimiento más cercano del paciente y que involucra a la familia, con una intervención psicosocial activa, ha demostrado ser eficaz para reducir el número de ingresos y mejorar el bienestar de los pacientes. ${ }^{30}$

\section{Necesidad de grupos de apoyo}

La mayoría de los familiares del grupo consideran importante que existan grupos de apoyo donde puedan compartir sus experiencias y dificultades con otras personas que viven una situación similar:

F7: "Yo siento que también necesito un grupo, donde me ayuden a entender a mi hijo y entenderme a mí misma, un grupo centrado en la enfermedad de mi hijo."

F6: "Necesitamos grupos [...] necesitamos dirección de psicólogos y de psiquiatras, necesitamos grupos donde nos podamos unir."

Estos grupos son muy importantes para formar redes sociales de apoyo entre familiares informados. Sin embargo, es necesario que exista un soporte por parte de los profesio- 
nales en el campo de la salud mental para que haya orientación y seguimiento.

\section{CONCLUSIONES}

Los familiares de pacientes con esquizofrenia, al ser los responsables del cuidado del enfermo en la comunidad, son quienes viven y constatan los diferentes tipos de crisis durante el transcurso de la esquizofrenia. A partir de lo expuesto por los familiares, podemos destacar tres principales situaciones de crisis: 1 . Primera crisis psicótica: cuando aparecen los primeros síntomas psicóticos en la fase prodrómica, los familiares desconocen lo sucedido y, por tanto, demoran el tratamiento del paciente porque no saben a dónde acudir para ser atendidos; 2 . Crisis familiar ante el diagnóstico y en el proceso de aceptación de la enfermedad: cuando aparece la enfermedad hay un impacto en los familiares que dificulta la aceptación del diagnóstico. Se producen cambios en la dinámica familiar por la convivencia cotidiana con el paciente y aumenta el estrés de los cuidadores, quienes en muchas ocasiones presentan otros trastornos, porque se dedican de tiempo completo al familiar enfermo sin recibir capacitación; 3. Recaídas subsecuentes y las continuas crisis: las recaídas, que se originan por diversas causas, dentro de las cuales se destacó la suspensión del medicamento. Respecto a los incisos 4. Deficiencias y 5. Necesidades en la atención, también se enumeran las necesidades a las que se enfrentan los familiares en las situaciones de crisis: necesidad de información, apoyo profesional e institucional para el manejo de crisis, necesidad de atención integral y de grupos de apoyo. Destaca la falta de información sobre los trastornos mentales en la población general y el manejo de la discriminación hacia los pacientes con esquizofrenia, razón por la cual se crea una barrera para el proceso de aceptación de la enfermedad. Además, de no tener conocimiento sobre las instituciones de salud donde son atendidas, difícilmente se va a buscar una atención oportuna. Los familiares requieren estar enterados de las medidas que deben tomarse en caso de una crisis o recaída, además de la necesidad de contar con servicios de urgencias que funcionen como verdaderos "centros de solución de crisis" con personal capacitado. Así mismo, se debe organizar la formación de programas de seguimiento, donde se brinde una atención más personalizada y se prevengan las recaídas. Por otra parte, es necesario implementar una asistencia integral donde se complemente el tratamiento farmacológico, psicológico, psicosocial y familiar. En particular, se debe incluir la psicoeducación ${ }^{35}$ para cubrir de esta manera las necesidades de los pacientes a largo plazo. Finalmente, es necesaria una adecuada atención a los familiares para mejorar su calidad de vida. ${ }^{16}$ Se ha observado que la participación familiar favorece la evolución del paciente, ya que la continuidad del tratamiento es más sostenible y el pronóstico se hace favorable. ${ }^{6}$

\section{AGRADECIMIENTOS}

Al Consejo Nacional de Ciencia y Tecnología de México (CONACyT) por el financiamiento de este estudio a través del proyecto 46569-H.

A todos los participantes del grupo de discusión por el tiempo y la información que nos brindaron y porque sin ellos no hubiera sido posible realizar la presente investigación.

\section{REFERENCIAS}

1. World Health Organization (2010). Schizophrenia. Consultado 2 noviembre de 2013, http://www.who.int/mental_health/management/ schizophrenia/en/

2. Organización Panamericana de la Salud. Informe sobre la salud en el mundo 2001. Salud Mental: nuevos conocimientos, nuevas esperanzas. Consultado junio de 2012: www.paho.org/Spanish/DD/PIN/saludmental_005.htm

3. Rascón ML, Caraveo J, Valencia M. Trastornos emocionales, físicos y psiquiátricos en los familiares de pacientes con esquizofrenia en México. Rev Inv Clin 2010;62(6):509-515.

4. Howard CW. Diccionario psicología. $10^{\mathrm{a}}$ reimp. México: Fondo de Cultura Económica; 1977.

5. Vírseda JA. Intervención familiar en crisis. Crisis y familia. Universidad Iberoamericana. Rev Depto Psic 1990(3):55-65.

6. Mascaró N. Crisis y contención. El grupo multifamiliar. AMSA. ASMR. Rev Inter On-line 2007:6(2):1-9.

7. Guimón J. Contención y crisis. ASMR Rev Inter On-line 2007:(6):1-11.

8. Rascón ML, Alcántara H. La familia como instrumento para reducir riesgos en la esquizofrenia. En: Alternativas terapéuticas en la esquizofrenia. Valencia M (comp) México: Ed. Herder; 2012; pp.305-330.

9. Maruottolo CB, Mascaró A, Guimón J. Factores clínicos en los pacientes en unidades de crisis AMSA. ASMR Rev Inter On-line 2007;(6):1-17.

10. Lemos-Giráldez S, Vallina-Fernández O. Dos décadas de intervenciones familiares en la esquizofrenia. Psicoth 2000:12(4):671-681.

11. Rascón ML, Díaz R, Jiménez JL, Reyes C. La necesidad de dar atención a los familiares responsables del cuidado de pacientes esquizofrénicos. Salud Mental 1997:20(Supl):55-64.

12. Rascón D, Valencia M. Los factores familiares de la esquizofrenia. En: Ortega-Soto H, Valencia-Collazos M (eds). Estado actual y perspectivas. Esquizofrenia. México: Colección del Instituto Nacional de Psiquiatría Ramón de la Fuente; 2001; pp. 399-458.

13. Budd R, Oles Gm, Hughes I. The relationship between coping style and burden in the careers of relatives with schizophrenia. Act Psych Scand 1998(4):304-309.

14. Muela JA, Godoy J. Programas actuales de intervención familiar en esquizofrenia. Psicoth 2001(13):1-6.

15. Murphy S, Irving CB, Adams CE. Crisis intervention for people with severe mental illnesses. Schizophr Bull 2012;38(4):676-677. Texto completo: http://schizophreniabulletin.oxfordjournals.org/content/38/4/676.full

16. Valencia M, Rascón ML, Quiroga H. Aportaciones de la investigación respecto al tratamiento psicosocial y familiar de pacientes con esquizofrenia. Salud Mental 2003;26:1-18.

17. De la Higara-Romero J. Psicoeducación familiar en la esquizofrenia: de los datos empíricos a la realidad clínica. Apuntes Psic 2005(23):275-292.

18. Díaz LR, Ortega H, Leaños C, Rodríguez MS et al. La rehabilitación integral del paciente esquizofrénico en México: el modelo del Instituto Nacional de Psiquiatría Ramón de la Fuente, Grupo III. Salud Mental 2005(28):9-19.

19. Alonso LE. La mirada cualitativa en sociología. Una aproximación interpretativa. Madrid: Fundamentos; 1998.

20. Krueger RA. 1994. Focus group. A practical guide for applied research. USA: SAGE; 1998. 
21. Davidson L. Narrativas de la esquizofrenia. El uso de la estructura narrativa en la investigación fenomenológica. Revista Psicoterapia 1995; 6(22-23):83-100.

22. Berenzon S, Medina-Mora ME, Lara MA. Servicios de salud mental: Veinticinco años de investigación. Salud Mental 2003(26):61-72.

23. Apiquián R, Fresán A, Ulloa R. El primer episodio psicótico. En: Ortega-Soto $\mathrm{H}$, Valencia-Collazos M (eds). Esquizofrenia. Estado actual y perspectivas. México: Colección del Instituto Nacional de Psiquiatría Ramón de la Fuente; 2001:250-299.

24. Ciruana M. Caminando hacia un nuevo modelo de atención multidisciplinaria de la esquizofrenia y otras psicosis. Papel de la atención primaria. Atención Primaria 2007:(39):125-126.

25. Fresán A, Apiquián R, Nicolini H, García-Anaya M. Association between violent behavior and psichotic relapse in schizophrenia: once more through the revolving door? Salud Mental 2007(30):25-30.

26. Apiquián R, Fresán A, García-Anaya M, Loyzaga C et al. El impacto de la duración de la psicosis no tratada en pacientes con primer episodio psicótico. Estudio de seguimiento a un año. Gac Méd México 2006:(142):113-120.

27. Valencia M, Ortega-Soto H, Rodríguez MS, Gómez L. Estudio comparativo de consideraciones clínicas y psicoterapéuticas en el tratamien- to biopsicosocial de la esquizofrenia. Primera parte. Salud Mental 2004:(27):47-53.

28. Velasco ML, Sinibaldi J. Manejo del enfermo crónico y su familia (sistemas, historias y creencias). México: Manual Moderno; 2001.

29. Creer C, Sturt E, Wykes T. The role of relatives. En: Wing long term community care: experience in a London brought. Psych Med Mon 1982;(Supl 2):29-39.

30. Lader M. What is relapse in schizophrenia? Int Clin Psych 1995(9):5-9.

31. Robinson D, Woerner G, Alvir MG Geisler JM et al. Predictors of treatment response from a first episode of schizophrenia or schizoaffective disorder. Americ J Psych 1999:(156):544-549.

32. Leff J, Vaughn $C$. The interaction of life events and relatives expressed emotion in schizophrenia and depressive neurosis. Brith J Psych 1980:(136):146-153.

33. Muela J, Godoy J. El estrés crónico en la esquizofrenia: La emoción expresada. REME. Rev Elec de Motivación y Emoción. 2001b;7(IV).

34. King S, Dixon M. Expressed emotion and relapse in young schizophrenia outpatients. Schic Bull 1999:(25): 377-386.

35. Zou H, Li Z, Nolan MT, Arthur D. Self-management education interventions for persons with schizophrenia: A meta-analysis. Int J. Ment Health Nurs 2013; 22(3):256-271.

Artículo sin conflicto de intereses 